\title{
Doença Trofoblástica Gestacional Complicada por Hemorragia
}

\author{
Hemorrhagic Complications of Gestational Trophoblastic Disease
}

Paulo Belfort, Luiz Gustavo Bueno, Carlos Eduardo Novaes, Jorge de Rezende

\section{RESUM0}

Objetivo: relatar três casos de doença trofoblástica gestacional, complicados por hemorragia grave, exigindo recurso cirúrgico, de fortuna, para obter a hemóstase.

Métodos: o estudo diz respeito a três pacientes. Em uma delas, paciente jovem, nulípara, de 27 anos foi realizada histerectomia total abdominal. Após a cirurgia tornou-se possivel prosseguir a quimioterapia e alcançar a remissão da doença. Outra paciente sangrava incoercivelmente mercê de extensa metástase vaginal, cuja hemostasia só foi conseguida mediante ligadura das artérias hipogástricas. A cura definitiva adveio com o prosseguimento da quimioterapia. Dois anos após o episódio, a paciente encontra-se novamente grávida. $O$ terceiro exemplo - mola persistente - apresentava tecido trofoblástico aderido ao segmento inferior do útero, do colo e do fundo de saco lateral direito da vagina, que sangrava copiosamente a cada tentativa de remoção cirúrgica: curetagem ou vácuo-aspiração. Conseqüente à múltipla manipulação invasiva da genitália, sobreveio grave processo infeccioso que evolveu para septicemia que a antibioticoterapia e a histerectomia (tardia) não conseguiram debelar, culminando com o óbito da paciente.

Resultado: as pacientes foram tratadas de modo excepcional: duas mediante histerectomia total abdominal e uma com ligadura das artérias hipogástricas. Daquelas submetidas à histerectomia uma sobreviveu e a outra veio a falecer de septicemia. A que foi tratada mediante ligadura das artérias hipogástricas alcançou remissão definitiva e, completado o seguimento, voltou a gestar, tendo gravidez de evolução normal.

Conclusão: conquanto doença trofoblástica gestacional tenha, de hábito, curso tranqüilo e remissão espontânea freqüente, complicações inusitadas exigem condutas radicais com desfecho nem sempre favorável.

PALAVRAS-CHAVE: Doença trofoblástica gestacional. Complicações hemorrágicas. Quimioterapia.

\section{Introdução}

A neoplasia trofoblástica gestacional (NTG mola hidatiforme completa e parcial) alcança remissão espontânea cerca de $80 \%$ das vezes ${ }^{1}$.

Centro de Neoplasia Trofoblástica Gestacional da "33

Enfermaria da Santa Casa da Misericórdia do Rio de Janeiro"

- (Serviço do Prof. Jorge de Rezende).

Correspondência:

Paulo Belfort

Avenida das Américas 3939, bloco 1, sala 216 - Barra da Tijuca

22630-003 - Rio de Janeiro - RJ

Telefone: (021) 3325-1481. FAX 3325-6643

e-mail: belfortbelfortp@aol.com
Evolvendo, entretanto, para doença trofoblástica gestacional (DTG), suas formas invasoras de alto risco, não obstante a terapêutica enérgica e oportuna nem sempre é a remissão obtida ${ }^{1-3}$.

As formas benignas da enfermidade - mola completa e parcial -, consoante aludido, têm curso clínico pelo geral discreto e curam espontaneamente, na maioria das vezes, após curto período de evolução.

Mercê do diagnóstico habitualmente precoce, como é de vezo na atualidade com o emprego da ultra-sonografia ${ }^{4,5}$, poucas vezes surgem complicações graves e incomuns a exigirem tratamen- 
to medicamentoso ou cirúrgico. Remissão espontânea é a regra ${ }^{6}$. Sangramento costumava ser, no passado já um tanto remoto, a principal complicação, a conta da infiltração miometrial (mola invasora) ou da malignização (coriocarcinoma). Em todas as eventualidades, entretanto, a quimioterapia tornou-se o tratamento de escolha, induzindo remissão completa na imensa maioria dos casos de estadiamentos iniciais $^{4,7}$, sendo o tratamento cirúrgico cada vez menos utilizado e, quase exclusivamente, nos casos graves, de risco, e em pacientes idosas e de prole constituída ${ }^{2}$.

As metástases pulmonares e vaginais da doença são as mais freqüentes, costumando regredir com a quimioterapia ${ }^{2}$. A presença de metástases vaginais ou de lesão uterina persistente e sangrante é complicação incomum a demandar medidas curativas igualmente insólitas.

Não sobrevindo remissão espontânea, cumpre estadiar a doença, a fim de tratar a paciente com regimes terapêuticos consagrados, pertinentes às suas diferentes fases. O estadiamento da doença trofoblástica gestacional é feito consoante proposição da Federação Internacional de Ginecologia e Obstetrícia (FIGO) e sancionada pela Organização Mundial da Saúde (OMS) em quatro graus: doença limitada ao útero; doença limitada à pelve; doença se estende aos pulmões (metástases pulmonares) e doença que se propaga a outras estruturas, além das mencionadas, principalmente cérebro e figado (Berkowitz e Goldstein, 1997) 4 .

Além de estadiar a doença cumpre estabelecer o risco, obtido mediante o sistema de contagem baseado em fatores prognósticos originalmente proposto por Bagshawe ${ }^{3}$, aprovado pela FIGO/ OMS e recentemente modificado pela Sociedade Internacional para o Estudo da Doença Trofoblástica (ISSTD) à ocasião do XI Congresso Mundial da especialidade realizado em Santa Fé (EUA), em novembro de 2001.

Refere a literatura atual ${ }^{5-7}$ e sanciona a experiência que, após o advento da ultra-sonografia, o diagnóstico precoce da mola permitiu a sua evacuação imediata, desta forma evitando ou reduzindo a mais freqüente manifestação clínica da doença - o sangramento genital. Vezes raras, entretanto, surgem complicações inusitadas que modificam e subvertem o quadro clínico, agravando intensamente o estado de saúde da paciente, exigindo medidas terapêuticas radicais, mutiladoras, por vezes heróicas, de fortuna que, quando adotadas tardiamente, não logram salvar a vida da paciente.

Refere-se o presente trabalho a três pacientes que no curso do seguimento pós-molar apresentaram grave complicação hemorrágica: duas sobreviveram e uma veio a falecer.

\section{Pacientes e Métodos}

São os casos aqui relatados oriundos do Centro de Neoplasia Trofoblástica Gestacional da $33^{\text {a }}$ Enfermaria da Santa Casa da Misericórdia do Rio de Janeiro que diagnostica, acompanha e trata os casos de DTG. É o seguimento de todas as pacientes com mola hidatiforme rigoroso, pontual e sistemático, permitindo a imediata identificação dos casos que não mostram tendência à remissão espontânea, mas necessitam tratamento para induzir remissão.

\section{Caso 1}

APLC, 27 anos, solteira, branca, carioca, doméstica, G1/P0, O, tipo sanguíneo $\mathrm{Rh}+$, última menstruação em 30 de agosto de 2000, apresentou menarca aos 15 anos, primeira relação sexual aos 21 anos. Relata cinco parceiros sexuais. Fez uso de contracepção hormonal oral, ininterruptamente, durante 6 anos, interrompendo-a com o objetivo de engravidar.

Em amenorréia desde 30 de agosto de 2000, com o diagnóstico de abortamento espontâneo, foi submetida a curetagem uterina (D/C), num hospital municipal do Rio de Janeiro, em 7 de novembro de 2000. No dia 10 de dezembro foi hospitalizada na Maternidade da Santa Casa, referindo dor tipo cólica no baixo ventre, sangramento transvaginal copioso e suspeita diagnóstica de neoplasia trofoblástica gestacional ao exame ultra-sonográfico. No mesmo dia foi submetida a novo raspado uterino. No curso do procedimento o cirurgião identificou perfuração uterina havida, presumivelmente, na curetagem anterior. A laparotomia exploradora realizada a seguir evidenciou lesão situada na parede anterior do útero, próxima ao fundo, logo suturada, procedendo também ao desfazimento de aderências dos órgãos pélvicos. Havia grande quantidade de sangue acumulado na cavidade abdominal. A paciente obteve alta, em bom estado, três dias depois, em 13 de dezembro de 2000.

Dois meses após, sem haver retornado ao Serviço, no dia 19 de fevereiro de 2001, comparece ao Ambulatório de NTG da 33를 Enfermaria apresentando útero aumentado de volume, hiperêmese, edema dos membros inferiores e nova ultra-sonografia indicativa de retenção de restos molares.

A dosagem de beta-hCG foi de $742.720 \mathrm{mUI} /$ $\mathrm{mL}$. Novamente internada, foi submetida a vácuoaspiração, com retirada de abundante material molar (cerca de $1.000 \mathrm{~mL}$ ), cujo exame histopatológico mostrou vilosidades volumosas, hidrópicas, intensa hiperplasia do trofoblasto e 
necrose. Radiografia dos pulmões realizada em 5 de março, mostrou a presença de metástases em ambos os pulmões.

Em 15 de março nova ultra-sonografia pélvica, transvaginal, comprovou restos de material molar na cavidade uterina e imagem de infiltração na parede anterior do útero; foi realizado mais um esvaziamento uterino ( 16 de março de 2001). Em 29 de março, havida drástica redução dos níveis de hCG (6.021 mUI/mL), houve, contemporaneamente, redução acentuada das imagens metastáticas pulmonares.

Sendo IIIB (baixo risco $=5$ ) o estadiamento da doença, foi iniciada quimioterapia com o regime EMA/CO. Foram administrados três ciclos desse regime. Em 29 de abril a paciente foi novamente hospitalizada, em caráter de urgência, por apresentar hemorragia vultosa, palidez cutâneomucosa intensa $(+++/ 4)$, febre de $40^{\circ} \mathrm{C}$, hemoglobina 5,6 g/dL e hematócrito 18,9\%. Foram transfundidas duas bolsas de concentrado de hemácias e administrado mais um ciclo do regime EMA/CO, além de antibioticoterapia.

Um mês após, em 30 de maio, a paciente foi uma vez mais hospitalizada a conta de novo e expressivo episódio hemorrágico. Apresentava, nessa ocasião, sinais de toxicidade da quimioterapia (alopécia, estomatite, anemia: hemoglobina 6,6\%, hematócrito $21,6 \%$, plaquetas $183.000 / \mathrm{mm}^{3}$ ). Realizou-se mais uma transfusão de 2 bolsas de concentrado de hemácias.

Revelou, a tomografia linear, nesse momento, ainda a presença de nódulos metastáticos em ambos os pulmões, exigindo mais um ciclo do regime EMA/CO. A despeito da quimioterapia, repetiram-se os episódios de sangramento, acentuando-se a queda do estado geral. Várias outras transfusões foram feitas, de plasma inclusive, mas as perdas sangüineas reiteradas impediam a melhora da saúde da paciente. Temendo-se o desenlace fatal, a despeito da pouca idade da enferma e da sua nuliparidade, decidiu-se operá-la, realizando histerectomia total abdominal e retirada de ambos os anexos, em 28 de junho de 2001. O pósoperatório transcorreu sem incidente, recebendo alta cinco dias após. O exame histopatológico da peça cirúrgica revelou lesão coriocarcinomatosa infiltrando o miométrio. Nos meses seguintes, a paciente evolui com redução dos níveis plasmáticos de hCG (Tabela 1). No momento em que é o relato feito (novembro, 2003), a paciente goza boa saúde, trabalhando, sua vida normalizada. Conquanto se recuse a voltar ao Centro Especializado da $33^{\text {a }}$ Enfermaria, sabe-se, mediante informações telefônicas, que a paciente mantémse hígida.
Tabela 1 - Dosagem seriada de gonadotrofinas coriônicas da paciente APLC durante o seguimento em que foi submetida a quimioterapia pelo regime $\mathrm{EMA} / \mathrm{CO}$ e a histerectomia total abdominal para conseguir hemostasia. Mola invasora que evolveu para coriocarcinoma.

\begin{tabular}{|c|c|c|}
\hline Data & & $\mathrm{hCG}$ (um/mL) \\
\hline \multicolumn{3}{|l|}{2001} \\
\hline Fevereiro & 22 & 742.720 \\
\hline \multirow[t]{3}{*}{ Março } & 5 & 593.280 \\
\hline & 19 & 118.760 \\
\hline & 29 & 6.024 \\
\hline Abril & 30 & 74,0 \\
\hline \multirow[t]{2}{*}{ Maio } & 12 & 0,2 \\
\hline & 31 & 1,8 \\
\hline \multirow[t]{2}{*}{ Junho } & 7 & 0,2 \\
\hline & 28 & 1,0 \\
\hline Julho & 12 & 0,3 \\
\hline Agosto & 9 & 1,5 \\
\hline Setembro & 12 & 1,8 \\
\hline Outubro & 11 & 2,5 \\
\hline Novembro & 22 & 2,5 \\
\hline \multicolumn{3}{|l|}{2002} \\
\hline Janeiro & $1^{0}$ & 1,3 \\
\hline Fevereiro & 21 & 2,6 \\
\hline
\end{tabular}

Três dosagens adicionais com intervalo mensal negativas.

\section{Caso 2}

MJLS, 37 anos, solteira, preta, GI/PO, tipo sangüíneo $\mathrm{O}, \mathrm{Rh}+$, marido $\mathrm{O}, \mathrm{Rh}+$. Última menstruação em 5 de outubro de 2000. Menarca aos 14 anos, primeira relação sexual aos 32 . Fez uso de contracepção hormonal oral durante vários anos, sem interrupção, até setembro de 2000, quando cessou o uso com o objetivo de engravidar.

Sentindo-se grávida, apresentando náuseas e vômitos, iniciou tratamento pré-natal em hospital público, sendo-lhe prescrita medicação sintomática e solicitados exames rotineiros de gravidez. Nos primeiros meses freqüentou, com regularidade, o ambulatório pré-natal, sem que qualquer anormalidade houvesse sido consignada. Em fevereiro de 2001, com aproximadamente 17 semanas de atraso menstrual, teve a primeira perda sangüínea, queixando-se, ao mesmo tempo, de forte cólica abdominal, sendo hospitalizada durante 48 horas no serviço onde vinha sendo acompanhada. A partir desse evento exibiu sucessivas e mais abundantes perdas sangüineas. Tal sintomatologia determinou diversas internações hospitalares até que, no início de abril, foi submetida a exame ultrasonográfico que diagnosticou mola hidatiforme.

Foi internada na 33 ${ }^{\text {a }}$ Enfermaria em 18 de abril de 2001, apresentando sangramento vaginal, útero grande para a idade gestacional, cistos 
tecaluteínicos de ambos os ovários e síndrome toxêmica. O esvaziamento uterino foi realizado no dia seguinte (19 de abril) mediante vácuo-aspiração. Recebeu alta, encaminhada para seguimento pós-molar no ambulatório especializado. As dosagens de gonadotrofina coriônica estão mostradas na Tabela 2.

Tabela 2 - Dosagem seriada de gonadotrofinas coriônicas da paciente MJLS antes e após histerectomia total abdominal e um ciclo do regime EMA/CO. A paciente faleceu de septicemia.

\begin{tabular}{lrrr}
\hline \multicolumn{1}{l}{ Data } & & hCG $(\mathrm{mU} / \mathrm{mL})$ \\
\hline 2001 & & & \\
& Abril & 4 & 411.880 \\
& Maio & 5 & 254.800 \\
& 10 & 307.010 \\
& 14 & 182.440 \\
& 21 & 166.160 \\
& Junho & 11 & 8.923 \\
\hline
\end{tabular}

Durante o mês seguinte apresentou sangramento profuso, sendo reinternada em 11 de maio com quadro infeccioso, sugestivo de infecção urinária. Ultra-sonografia em 14 de maio mostrou útero volumoso contendo restos molares.

Em 16 de maio, tentativa de esvaziamento uterino provocou hemorragia vultosa, levando a paciente a quadro de choque. Havia, com efeito, lesão no segmento inferior do útero, extensiva ao colo e fundo de saco lateral direito da vagina, de onde provinha o sangramento. A hemóstase foi obtida após sutura em massa da aludida região. No local suturado os tecidos necrosaram. Em 5 de junho a ultra-sonografia revelava o mesmo conteúdo molar na cavidade uterina, ocupando a região ístmica e parte do canal cervical. O quadro clínico manteve-se inalterado, daí em diante.

Durante o período mencionado, desde a tentativa frustrada de esvaziamento uterino, a paciente teve diversos episódios de sangramento profuso, exigindo tamponamento, forma única de debelar a perda sangüinea. Por esse motivo, após generosa transfusão de sangue a paciente foi submetida a um ciclo do regime EMA/CO, de 31 de maio a 7 de junho, na tentativa de eliminar o tecido molar residual. Em 29 de junho, ostentando quadro de infecção grave, a despeito da ampla cobertura antibiótica e persistindo a incontrolável hemorragia, como medida de salvação foi submetida a histerectomia total abdominal com retirada bilateral dos anexos e do terço superior da cúpula vaginal. A paciente já se encontrava septicêmica. Foi transferida para a UTI, vindo a falecer poucos dias após, em 4 de julho de 2001.

\section{Caso 3}

VSOS, 24 anos, casada, branca, GIII/P II, tipo sangüíneo $\mathrm{A}, \mathrm{Rh}+$, marido $\mathrm{A}, \mathrm{Rh}+$. Data da última menstruação 15/3/2001. Menarca aos 9 anos, primeira relação sexual aos 15 anos. Duas gestações anteriores normais, a termo, e partos vaginais. A primeira gravidez foi aos 17 anos (1994) e a segunda, aos 19 anos (1996). Após atraso menstrual, sem saber-se grávida, iniciou sangramento em 1 de maio (7 semanas), durante 5 dias. Teve perdas sangüíneas irregulares ao longo de todo o mês de maio. No início de junho, percebeu indícios de gravidez, realizando teste que resultou positivo (7 de junho). Procurou médico, a seguir, sendo solicitado exame ultra-sonográfico, que revelou a mola.

Foi atendida no Centro de NTG da $33^{a}$ Enfermaria no dia 19/6, sendo hospitalizada e submetida a vácuo-aspiração no dia 21 de junho. Uma semana após (28 de junho), foi reinternada, apresentando intensa hemorragia proveniente de metástase vaginal que ocupava toda a sua parede lateral direita. Com o diagnóstico de doença trofoblástica estádio IIB (baixo risco $=3$ ), foi submetida a dois ciclos do regime EMA/CO: o primeiro de 28 de junho a 5 de julho e o segundo de 12 a 19 de julho. Três dias após o término do segundo ciclo de quimioterapia, a paciente foi reinternada exibindo profuso sangramento proveniente da metástase vaginal só coibido mediante maciço tamponamento local.

Esboçou-se quadro infeccioso que foi prontamente debelado com a antibioticoterapia instituída. Prosseguiram as intensas perdas sangüineas, fazendo a paciente tangenciar o estado de choque e exigindo numerosas transfusões de sangue. $O$ quadro parecia tornar-se irreversível, diante da impossibilidade de retomar a quimioterapia em virtude do precário estado de saúde da paciente, até que se decidiu - como medida heróica - realizar ligadura das artérias hipogástricas, efetuada em 9 de agosto. Daí em diante o sangramento cessou, sendo retomada a quimioterapia. Três ciclos adicionais do regime EMA/CO foram realizados de 17 a 24 de agosto, de 3 a 10 de setembro e de 24 de setembro a 1 de outubro de 2001.

Nesse entretempo, a radiografia dos campos pleuro-pulmonares mostrou, em 15 de agosto, pequeno nódulo metastático localizado no terço médio do pulmão direito, que regrediu após o término da quimioterapia. O exame pélvico realizado em 30 de agosto revelou a quase completa regressão da lesão vaginal. Os níveis de hCG baixaram acentuadamente, consoante é mostrado na Tabela 3. 
Tabela 3 - Dosagem seriada de gonadotrofinas coriônicas da paciente VSOS durante o seguimento em que foi submetida a quimioterapia pelo regime EMA/CO e a ligadura das artérias hipogástricas para conseguir hemostasia da metástase vaginal sangrante.

\begin{tabular}{|c|c|c|}
\hline Data & & $\mathrm{mUI} / \mathrm{mL}$ \\
\hline \multicolumn{3}{|l|}{2001} \\
\hline \multirow[t]{2}{*}{ Junho } & 21 & 1.730 .400 \\
\hline & 28 & 323.880 \\
\hline \multirow[t]{2}{*}{ Julho } & 9 & 69.460 \\
\hline & 26 & 783 \\
\hline \multirow[t]{2}{*}{ Agosto } & 10 & 25,9 \\
\hline & 30 & 10,7 \\
\hline \multirow[t]{2}{*}{ Setembro } & 17 & 5,8 \\
\hline & 24 & 4,0 \\
\hline \multirow[t]{2}{*}{ Outubro } & 11 & 3,2 \\
\hline & 22 & 1,9 \\
\hline Novembro & 12 & 3,3 \\
\hline \multicolumn{3}{|l|}{2002} \\
\hline Fevereiro & 4 & 1,7 \\
\hline Março & 21 & 0,5 \\
\hline
\end{tabular}

Oito dosagens adicionais com intervalo mensal em 2002-2003 negativas.

A paciente se encontra, no momento em que o texto é redigido (novembro de 2003), em remissão definitiva, clínica e hormonal, e novamente grávida, havendo sido sua última menstruação em 23 de fevereiro de 2003.

\section{Discussão}

O diagnóstico da mola hidatiforme vem sendo feito cada vez mais cedo, a conta do emprego quase sistemático da ultra-sonografia no início da gravide ${ }^{8}$. A descoberta precoce da mola poderia propiciar medidas terapêuticas enérgicas, eficientes, capazes até de evitar a evolução invasiva e/ou maligna da doença.

Os três casos relatados mostram epílogo satisfatório em dois e término fatal em um, deixando entrever que algumas vezes não é possível seguir o protocolo estabelecido e obter resultados sistematicamente alvissareiros. Analisando criticamente, porém, os três exemplos, evidencia-se que se houvéssemos atuado mais agressivamente e com mais antecedência no caso da paciente que faleceu, tirando-lhe o útero, ligando-lhe as artérias hipogástricas ou promovendo embolização dos vasos sangrantes, talvez o resultado fosse diferente. Cumpre salientar que as duas sobreviventes ostentavam condições de saúde e de nível socioeconômico mais favorável, o que talvez lhes haja conferido maior resistência orgânica e melhor resposta ao tratamento instituído.

Outro elemento relevante foi, possivelmente, a idade das pacientes; as sobreviventes tinham 24 e 27 anos, respectivamente, enquanto a que morreu tinha 37. Schorge et al. ${ }^{7}$ consignam que riscos e complicações da mola completa são, em relação a mulheres jovens, segundo Parazzini, citado pelos autores, duas vezes mais freqüentes em mulheres com mais de 35 anos e 7,5 maior naquelas com mais de 40 anos.

Xiang et al. ${ }^{9}$ analisaram retrospectivamente 27 casos de NTG tratados mediante cirurgia de urgência, entre 1985 e 1996. Dezessete dos 27 casos foram diagnosticados como coriocarcinoma e 10 como mola invasora. Desse total, 16 pacientes foram submetidas a histerectomia total à conta de perfuração uterina ou de sangramento maciço; quatro outras sofreram ooforectomia unilateral, motivada por torção de cistos tecaluteínicos; além de cirurgias realizadas em três pacientes para remoção de metástases cerebrais, duas outras exibiram hemorragia genital grave, quase fatal, proveniente de metástase vaginal, embora os autores não mencionem o tipo de tratamento realizado. Referem ainda que 17 pacientes não foram submetidas a quimioterapia antes de serem operadas, seis recebendo um ciclo de tratamento pré-operatório. Vinte e três pacientes submetidas a vários ciclos de quimioterapia com múltiplos agentes, após a cirurgia, alcançaram completa e definitiva remissão. Concluem afirmando que a cirurgia desempenha importante papel no tratamento de pacientes com DTG, em situações emergenciais (e.g., hemorragias quase fatais) e que o diagnóstico precoce e a imediata adoção da quimioterapia talvez tornassem a cirurgia desnecessária.

Vardar et al. ${ }^{10}$, da Turquia, avaliaram 12 pacientes com metástase vaginal, em 75 casos de DTG, visando a determinar o prognóstico e as implicações terapêuticas. Duas pacientes receberam o regime MAC III (5 e 7 ciclos), quatro pacientes receberam o regime EMA/CO (2 a 11 ciclos) e seis foram tratadas com monoquimioterapia pelo metotrexato. Eis os resultados obtidos: três pacientes que receberam exclusivamente metotrexato não responderam ao tratamento, sendo transferidas para o regime EMA/CO. O índice total de sobrevida foi 91,6\%. Dizem, concluindo, que a presença de extensas metástases vaginais deveria constituir fator de alto risco e suas portadoras tratadas por regimes quimioterápicos de múltiplos agentes.

Guedes et al. ${ }^{11}$ relatam caso exitoso de ligadura das artérias hipogástricas em paciente com câncer avançado do colo uterino (estadiamento IIIb), exibindo hemorragia profusa e incontrolável após as medidas clínicas adotadas. Propõem que o procedimento cirúrgico de salvação seja adotado em situações emergenciais como descolamento prematuro da placenta acompanhado de atonia uterina, gravidez abdominal com hemorragia do leito placentário, laceração do canal vaginal provocando sangramento incoercível, placenta acreta e em qualquer situação em que haja sangramento 
genital abundante, quando os métodos convencionais não logrem obter hemostasia.

Yingna et al. ${ }^{12}$, de Pequim, analisaram retrospectivamente 51 pacientes com metástases vaginais das quais $8,6 \%$ estavam relacionadas a coriocarcinoma e $4,1 \%$ a mola invasora. Dezoito pacientes apresentaram hemorragia e ruptura da lesão, sendo todas tratadas mediante associação de vários agentes com 5-fluoracil. Dezesseis pacientes necessitaram tamponamento maciço da lesão para coibir o sangramento, sendo três submetidas a embolização angiográfica seletiva. Após a quimioterapia as metástases vaginais regrediram. Quarenta e quatro pacientes obtiveram remissão completa, sendo seguidas periodicamente sem evidência de recorrência. Afirmam os autores, em conclusão, que a presença de múltiplas e extensas metástases vaginais situa as pacientes em alto risco de apresentar hemorragia maciça e que a embolização angiográfica constitui método emergente de valia para controlar o sangramento decorrente de tumores vaginais.

Goldberg et al. ${ }^{13}$ descrevem 5 casos de DTG com metástases vaginais hemorrágicas, nas quais foi necessário, para coibir o sangramento, sutura das lesões sob anestesia. Uma paciente necessitou embolização arterial seletiva. Afirmam, à semelhança dos demais autores, que a presença de metástases vaginais sangrantes constitui fator de alto risco, exigindo que tais pacientes sejam tratadas, desde o começo da doença, mediante regime quimioterápico de vários agentes.

Cumpre, finalizando, lembrar que em situações graves, emergenciais, como as ora relatadas, além das medidas convencionais e provisórias visando a hemostasia (e.g., ergóticos, ocitocina por via sistêmica, tamponamento, etc.) deve-se ter em mente que a sobrevida das pacientes dependerá, muita vez, de atitudes corajosas, de fortuna, cirúrgicas e, não raro, mutiladoras.

\section{ABSTRACT}

Purpose: to report three cases of gestational trophoblastic disease with intense hemorrhagic complications, in which exceptional surgical procedures were used to obtain hemostasis.

Methods: the study comprised three patients: the first, a young woman, 27 years old, nullipara, was submitted to total abdominal hysterectomy and, thereafter, to chemotherapy until remission was achieved. Another patient bled from an extensive vaginal metastasis that could only be treated with hypogastric arterial ligation. Definitive sustained remission was obtained after chemotherapy. Two years after the episode, the patient achieved a new, normal pregnancy. The third patient, with persistent trophoblastic disease, presented a mass of molar tissue within the uterine inferior segment and cervix, extending to the right vaginal cul-de-sac, heavily bleeding at each attempt of surgical removal, whether by sharp or suction curettage. As a consequence of the invasive maneuvers she became seriously infected with sepsis; although being submitted to intensive antibiotic therapy and total abdominal hysterectomy she died a few days later.

Results: of the two patients who were submitted to total abdominal hysterectomy, one survived and the other died of septicemia. The third patient, who was submited to hypogastric arterial ligation, had a favorable outcome and achieved a new and normal pregnancy.

Conclusion: albeit gestational trophoblastic disease usually has an undisturbed course and spontaneous remission, unexpected complications may demand radical approaches leading sometimes to unfavorable results.

KEYWORDS: Gestational trophoblastic disease. Hemorrhagic complications. Chemotherapy.

\section{Referências}

1. Greenfield AW. Gestational trophoblastic disease: prognostic variables and staging. Semin Oncol 1995; 22:142-8.

2. Wright JD, Mutch DG. Treatment of high-risk gestational trophoblastic tumors. Clin Obstet Gynecol 2003; 46:593-606.

3. Bagshawe KD. Risk and prognostic factors in trophoblastic neoplasia. Cancer 1976; 38:1373-85.

4. Berkowitz RS, Goldstein DP. Presentation and management of molar pregnancy. In: Hancock BW, Newlands ES, Berkowitz RS, editors. Gestational Trophoblastic Disease. $1^{\text {st }}$ ed. London: Chapman \& Hill; 1997. p. 127-42.

5. Coukos G, Makrigiannakis A, Chung J, Randall TC, Rubin SC, Benjamin I. Complete hydatidiform mole: a disease with a changing profile. J Reprod Med 1999; 44:698-708.

6. Soper JT. Staging and evaluation of gestational trophoblastic disease. Clin Obstet Gynecol 2003; 46:570-8.

7. Schorge JO, Goldstein DP, Bernstein MR, Berkowitz RS. Recent advances in gestational trophoblastic disease. J Reprod Med 2000; 45:692-700.

8. Shapter AP, McLellan R. Gestational trophoblastic disease. Obstet Gynecol Clin North Am 2001; 28:805-17.

9. Xiang Y, Yang X, Zhang L, Song H. Evaluation of emergency surgery in gestational trophoblastic tumours. Zongguo Yi Xue Ke Xue Yuan Xue Bao 1997 ; 19:369-72.

10.Vardar MA, Altintas A, Demir SC. Gestational trophoblastic disease: the significance of vaginal metastases. Eur J Gynaecol Oncol 2000; 21:184-6.

11.Guedes RP, Nogueira ST, Tomaz G, et al. Ligadura das artérias hipogástricas para controle de hemorragia profusa por câncer avançado do colo uterino. GO Atual 1997; 6:49-50.

12.Yingna S, Yang X, Xiuyu Y, Hongzhao S. Clinical characteristics and treatment of gestational trophoblastic tumor with vaginal mestastasis. Gynecol Oncol 2002; 84:416-9.

13. Goldberg GL, Yon DA, Bloch B, Levin W. Gestational trophoblastic disease: the significance of vaginal metastases. Gynecol Oncol 1986; 24:155-61.

Recebido em: 8/12/2003 Aceito com modificações em: 12/7/2004 